\title{
The impact of accurate distances to dwarf elliptical galaxies on cosmology
}

\author{
Helmut Jerjen
}

Research School of Astronomy and Astrophysics, Australian National University, Australia email: jerjen@mso.anu.edu.au

\begin{abstract}
The Surface Brightness Fluctuation method has been shown to be a powerful distance indicator for dwarf elliptical galaxies to very low surface brightness levels. It is applicable to stellar systems that are out of reach for classical indicators requiring resolved stellar populations such as the tip magnitude of the red giant branch. I briefly discuss a few results from recent SBF studies of dEs to demonstrate the significance of the SBF method to address longstanding issues related to cosmography, dark matter in galaxy groups, substructures in clusters, and the discrepancy between the mass function of collapsed objects and the faint end of the galaxy luminosity function. For the analysis of the large number of galaxy images that need to be processed as part of such SBF studies we are currently developing a fast, semi-automatic reduction pipeline that will be made readily available to the astronomical community.
\end{abstract}

Keywords. galaxies: clusters: individual (Virgo)-galaxies: elliptical and lenticular-galaxies: dwarf galaxies: distances and redshifts, galaxies: luminosity function, mass function

\section{Motivation}

The ability to spatial locate newly detected dwarf galaxy candidates is of fundamental importance as it enables us among others to allocate them to an environment and to measure physical quantities such as their luminosity, mass, and star formation history. For instance any dwarf galaxy that can be found either in or close to the Local Group is of greatest interest. Such stellar systems are relatively rare and due to their proximity represent prime targets for detailed stellar population and dark matter studies. That explains why the scientific interest in Andromeda IV, discovered during van den Bergh's (1972) search for dwarf spheroidal companions to M31, was initially high but depreciated quickly when a radial velocity and a tentative distance measurement revealed 28 years later (Ferguson, Gallagher \& Wyse 2000) that it actually is a background irregular galaxy well outside the confines of the Local Group.

But before a distance can be measured the dwarf galaxy needs to be detected first. A modern and probably the most promising way to find gas-rich dwarf irregular (dIrr) galaxies in the local Universe is via deep, blind HI surveys using newly developed multibeam instruments at radio telescopes. Because low luminous and low surface brightness dIrrs have increasingly large HI mass fractions (e.g. Warren et al. 2004), simultaneous detection and distance measurement via redshift remains possible at $21 \mathrm{~cm}$ even in extreme cases like DDO154 (Krumm \& Burstein 1984; Carignan \& Freeman 1988) or ESO215G?009 (Warren et al. 2004) where only an elusive stellar component is present, embedded in an extended disk of neutral hydrogen that has not been involved in the galaxy's starformation processes.

Evidence for a high detection completeness with this search method is provided by the HI Parkes All-Sky Survey (HIPASS, Barnes et al. 2001). Perhaps somewhat surprising, HIPASS found no genuine dark galaxies, ie. extragalactic HI clouds or dark 
matter halos filled with $H I$ but no stars above the detection limit of $M_{H I}>10^{7} M_{\odot}$. All but a very small number of the $\sim 6000$ HIPASS detections in the surveyed velocity range $-700 \mathrm{~km} \mathrm{~s}^{-1}<v_{\odot}<12,700 \mathrm{~km} \mathrm{~s}^{-1}$ could be associated with stars (Koribalski et al. 2004, Doyle et al. 2005). The few exceptions are tidal features (e.g. Ryder et al. 2001, Oosterloo et al. 2004) or high velocity clouds (Kilborn et al. 2000).

Finding dwarf elliptical (dE) galaxies is by nature a more difficult task. Karachentsev with collaborators and many others identified large numbers of $\mathrm{dE}$ candidates in the direction of nearby groups and clusters over the last decade using primarily wide-field photographic plates. The challenge remains to measure distances to all of them to separate the wheat from the chaff. Unlike in the case of the dIrrs, the absence of gas in dEs prevents a relative simple distance estimate via redshift. Moreover, the large scatter in well-known empirical scaling relations based on integral galaxy parameters such as the surface brightness-luminosity (Binggeli \& Cameron 1991) or the shape parameterluminosity relation (Binggeli \& Jerjen 1998) makes them unsuitable for individual distance measurements.

That is why in the last decade, a formidable observational effort has been devoted to derive distances to dEs by using the tip magnitude of the red giant branch (TRGB), a powerful Pop II distance indicator (Karachentsev et al. 2004 and references therein). But the requirement of accurate photometry for individual stars 1-2 magnitudes below the tip sets a practical application limit at $\approx 5 \mathrm{Mpc}$. TRGB measurements for galaxies beyond that limit become quickly very costly and time consuming as was demonstrated by Harris et al. (1998) who spent 9 hours with the HST to obtain the TRGB distance for one single dE (IC3388) in the Virgo cluster.

Because of this limitation, a relatively new distance indicator the so-called surface brightness fluctuation (SBF) method has received increasing attention in recent years. The method quantifies the statistical pixel-to-pixel variation of star counts across a galaxy image with the major technical advantage of working on unresolved stellar populations. Ground-based CCD images with relative short integration times can be used instead of high resolution space-based observations which makes the SBF method extremely efficient. Distance measurements for early-type galaxies far beyond the reach of any other distance indicator become possible.

The theoretical framework of the SBF method was developed by Tonry \& Schneider (1988) with one of the main aims to study the distribution and kinematical properties of large-scale structure outlined by luminous giant ellipticals in the local Universe (e.g. Tonry et al. 2001). While SBF applications were most exclusively focussed on these high surface brightness systems, Jerjen and collaborators (Jerjen, Freeman \& Binggeli 1998, 2000; Jerjen et al. 2001, Rekola, Jerjen \& Flynn 2005) demonstrated that the method works equally well for low surface brightness dEs as faint as $\mu_{\mathrm{B} \text {,eff }}=$ $26 \mathrm{mag} \operatorname{arcsec}^{-2}$ and $M_{B}=-10 \mathrm{mag}$. With this possibility to measure distances to large numbers of dEs easily, including the newly identified $\mathrm{dE}$ candidates, a special opportunity arises to make progress in areas most relevant to near-field cosmology including

- Mapping the gravitational centers in the Supergalactic plane and Local supercluster.

- Substructures and dark matter contents of galaxy clusters.

- $\mathrm{dE}$ density in the local Universe.

- Shape of the galaxy luminosity function to the lowest luminosities.

\section{SBF calibration}

As the strength of the SBF signal and the derived fluctuation magnitude $\bar{m}$ depend not only on the distance of the galaxy but also on its stellar composition, the calibration of 


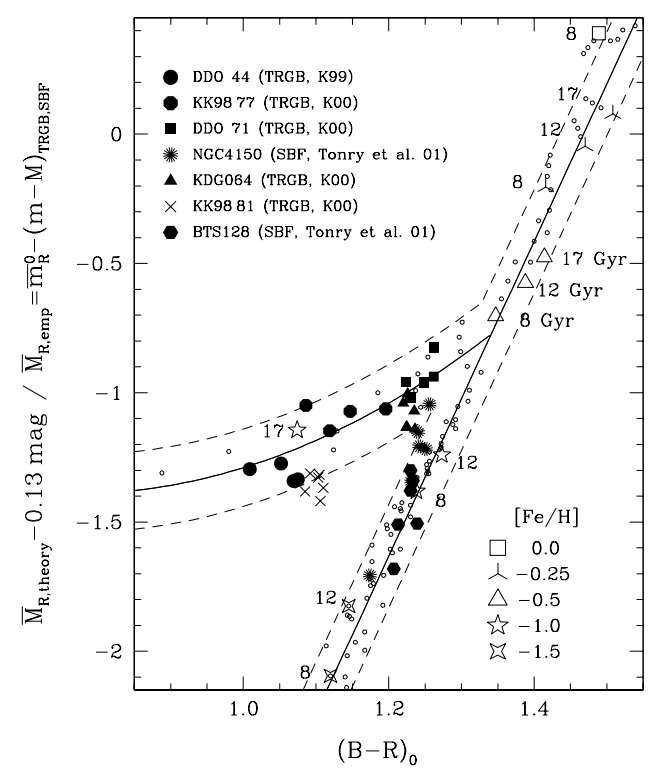

Figure 1. The SBF calibration diagram for the $R$-band. The open symbols are the $\left(B-R, \bar{M}_{R}\right)$ values for 170 synthetic stellar populations based on Worthey (1994) models and Padova isochrones (Bertelli et al. 1994). An offset correction of $0.13 \mathrm{mag}$ is applied to the theoretical values to match the empirical results from $36 \mathrm{SBF}$ fields measured in 7 nearby dwarf galaxies with independent TRGB and SBF distances (filled symbols). The solid lines represent the best analytical functions describing the loci of the models while the dashed lines indicate the \pm 0.15 mag uncertainty bands.

the method must take into account the basic properties of the stars. For a first theoretical calibration of the SBF method in the $R$-band, Jerjen et al. (1998, 2000) studied the effect of different mixtures of ages and metallicity as well as the starformation history on the observable quantities, fluctuation magnitude $\bar{M}_{R}$ and $B-R$ colour. For that purpose, Worthey's (1994) models combined with Padova isochrones (Bertelli et al. 1994) were employed to simulate a range of synthesis stellar populations with two starformation bursts to make allowance for the variety of starformation histories observed among Local Group dEs/dSphs (Grebel 2001). The age of the first burst was set at 8, 12, or 17 Gyr with a metallicity in the range from -2.0 to 0 . We added a second population, 5 Gyr old and of solar metallicity, that contributed $0,10,20$, or $30 \%$ in mass to the entire population. In all cases we assumed a Salpeter IMF. The $\left(B-R, \bar{M}_{R}\right)$ values for a total of 170 synthetic stellar populations were calculated.

The theoretical predictions were then tested with 36 SBF measurements in seven nearby galaxies (Jerjen et al. 2001, Rekola, Jerjen \& Flynn 2005) for which independent distances were available (Karachentsev et al. 1999, 2000; Tonry et al. 2001). Good agreement was found after a systematic offset correction of $0.13 \mathrm{mag}$ was applied to the theoretical values. These corrected $\left(B-R, \bar{M}_{R}\right)$ data points are shown in Fig. 1, indicated by the various open symbols. The two lines are best analytical fits of the empirically anchored theoretical data and represent the calibration equations for the SBF method in the $R$-band currently available (Jerjen et al. 2001). The typical overall uncertainty in a SBF distance measurement is $\approx 10 \%$ which includes the intrinsic scatter of the Worthey+Padova model values and an uncertainty of 0.1 mag in the TRGB zero point. 
Calibrations for other filter sets have been established by various authors (e.g. $V, I$ filters, Tonry et al. 1997, Mieske \& Hilker 2003; SDSS g, z filters, Mei et al. 2005).

\section{Some SBF applications of cosmological relevance}

\subsection{D-mapping of cluster cores}

There is a growing interest in the structural analysis of galaxy clusters to evaluate the importance of external mechanisms driving galaxy evolution in different kinds of environments, from cluster cores to their outskirts. In this context the characterization of the physical conditions at the immediate location of a cluster galaxy (distance) and the galaxy's dynamical status (velocity) are essential quantities.

Methods for detecting cluster substructure classically resort to projected galaxy positions and redshifts. But as redshifts are generally available only for high surface brightness galaxies, Es, S0s, and spirals where the latter are no genuine cluster galaxies and the number of Es+S0s in a cluster is relatively small, samples tend to be dominated by late-type galaxies that are infalling at a late stage of the cluster evolution and thus are kinematically distinct from genuine cluster galaxies. Consequently, these studies preferencially probe substructures in the cluster outskirts which are due to directional, inhomogenous galaxy aggretion along the larger-scale filaments a cluster is embedded in.

The detection of substructures/group remnants in the densest cluster regions where early-type galaxies dominate is more difficult as this is hampered by the small number of available giant ellipticals and the general lack of redshift information for dEs, the largest galaxy population that resides long enough in the cluster to trace the gravitational potential. Accurate distances of a sufficient number of early-type galaxies are indispensable for such studies.

In a pilot project, Jerjen, Binggeli \& Barazza (2004) measured SBF distances to 16 luminous dEs and combined them with SBF distances to giant ellipticals from the literature (Neilsen \& Tsevtanov 2000, Tonry et al. 2001) to trace the gravitational centers of the Virgo cluster along the line-of sight. The sample dEs showed a substantial spread in distances ranging from 14.9 to $21.3 \mathrm{Mpc}$ (see Fig. 2) which confirmed that the Virgo cluster core has an elongated shape, consistent with the previous findings of Neilsen \& Tsvetanov (2000), West \& Blakeslee (2000) and Arnaboldi et al. (2000). However, somewhat unexpected was that this seemingly dynamically relaxed system of early-type galaxies were clumped in two major concentrations at $(m-M)=31.02 \mathrm{mag}(15.8 \mathrm{Mpc})$ and at $31.33 \mathrm{mag}(18.5 \mathrm{Mpc})$ with a number ratio of $3: 1$ (Fig. 2). An adaptive kernel analysis of the distribution found that these clumps are significance at the $99 \%$ and $89 \%$ level, respectively, and that there might be even a minor third clump of galaxies at $\approx 31.55$ mag.

By combining the newly obtained SBF distances with redshift information from the literature the long-standing mystery around the four highly blueshifted dE galaxies VCC810, 815, 846, and 928 could be solved. The distance-velocity map (Fig. 2) revealed that these systems are part of the second clump approximately $2.7 \mathrm{Mpc}$ at the back of the major galaxy concentration at $15.8 \mathrm{Mpc}$. Together with M86, these dEs constitute a dynamically independent group infalling into the cluster from the backside for the first time. These dwarf systems might have been late-type disk galaxies which, by gravitational interactions with the ICM and other cluster members, lost their gas and transformed into the spheroids we see today (Mao \& Mo 1998, Moore et al. 1998, Mastropietro et al. 2005). Therefore, they present prime targets for follow-up studies to search for HI cloud remnants and structural features as have been already found in other Virgo dEs (Jerjen, 

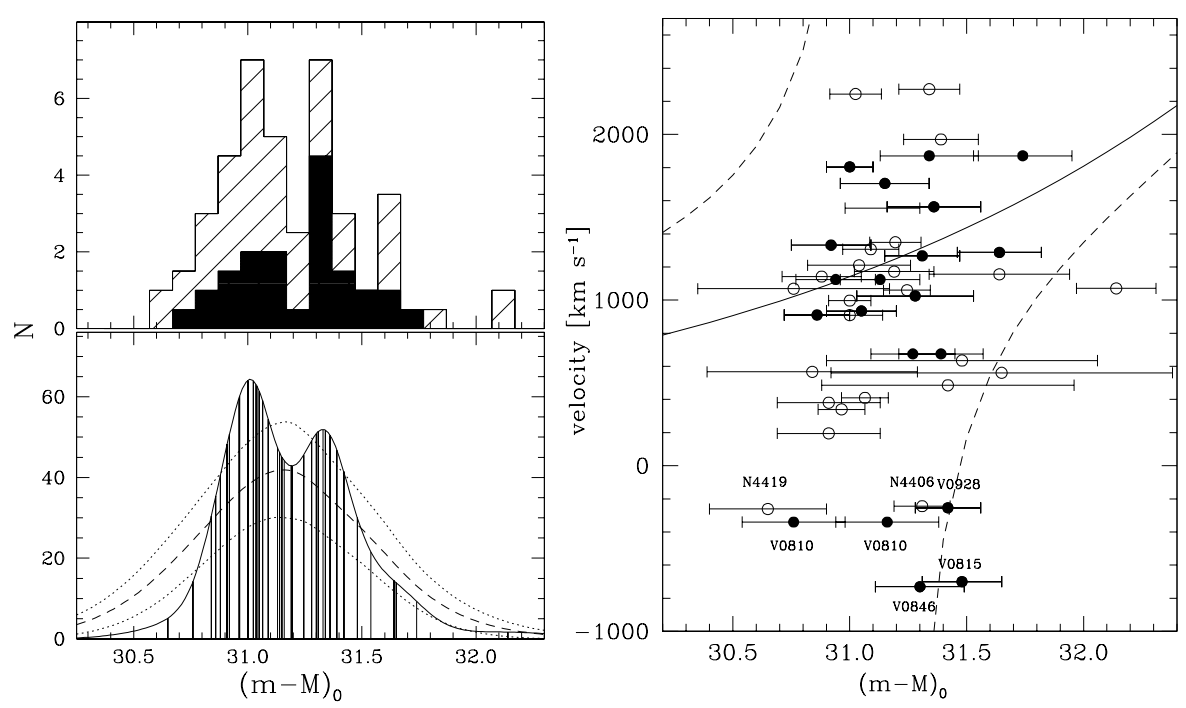

Figure 2. (Left-top): distance distribution of 41 early-type dwarf (solid histogram) and giant (hatched histogram) galaxies in the Virgo cluster. (Left-bottom): the same distribution based on the adaptive kernel method allows the quantification of the apparent bimodal structure. (Right): the distance-velocity plot (black: dEs, circle: Es) identifies the four highly blueshifted Virgo galaxies VCC810, 815, 846, and 928 as a separate group centered around M86 infalling from the backside of the cluster. VCC810 is shown twice to indicate its two possible SBF distances (For more details see Jerjen, Binggeli \& Barazza 2004).

Kalnajs \& Binggeli 2000; Barazza, Binggeli \& Jerjen 2002) and to test environmentally induced transformation processes such as the harassment scenario developed by Moore et al. (1998).

More results on the spatial distribution of Virgo Es and dEs can be expected from the ACS-Virgo Survey (Côté et al., this volume) where SBF distances to $\approx 100$ early-type galaxies will be reported. The ACS Survey is still a targeted survey. To handle the even larger volumes of CCD images of cluster galaxies produced by ground-based wide-field camera surveys at telescopes like Subaru, VISTA, CFHT, and VST in the near future, we are currently developing a fast SBF reduction package (Dunn \& Jerjen, this volume).

\subsection{Toward a distance-based galaxy luminosity function}

It is becoming increasingly evident that there are fewer dwarf galaxies per giant galaxy observed in the local Universe than anticipated by the Cold Dark Matter (CDM) hierarchical clustering models (Klypin et al. 1999; Moore et al. 1999; Trentham \& Tully 2002). This problem may well have a complex solution: variations on CDM cosmology, such as Warm Dark Matter (Bode et al. 2001) or Self-Interacting Dark Matter (Spergel \& Steinhardt 2000). Alternatively, there is no shortage of astrophysical mechanisms that can diminish the accumulation of baryons in low mass potential wells: long cooling times for primordial gas in small halos (Haiman et al. 1996), galactic winds driven by supernovae and hot stars (Dekel \& Silk 1986), or pressure support against collapse of the intergalactic plasma after reionization (Thoul \& Weinberg 1996; Gnedin 2000).

The present situation is confused because there are a multitude of reasons why the faint end of the galaxy luminosity function that is completely governed by the dwarf galaxies (Binggeli, Sandage \& Tammann 1988) could deviate from the simple CDM theory expectation. Progress on this important problem is currently limited by observations, not theory. Most recent studies of the galaxy luminosity function in Virgo (Phillipps et al. 

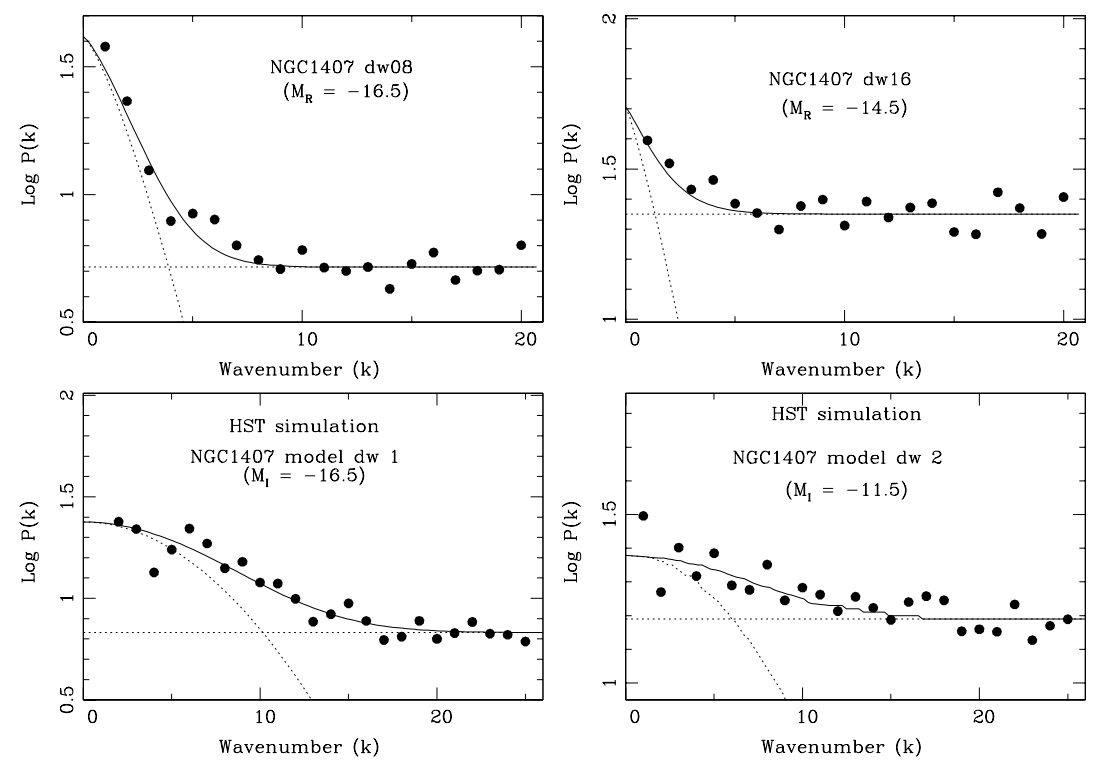

Figure 3. The top panels show the $R$-band power spectra of two dwarf galaxies at the the NGC1407 distance of $25 \mathrm{Mpc}$ obtained with 12 minute exposures in 1" seeing with the $8 \mathrm{~m}$ Subaru Telescope. The bottom panels show simulated $I$-band power spectra of NGC1407 dwarfs as would be obtained with 20 minute exposures with HST/ACS. The absolute magnitudes of the targets are indicated in the panels. The brighter and fainter simulated targets have central surface brightnesses of 22.5 and $25 \mathrm{I} \mathrm{mag} \operatorname{arcsec}^{-2}$ respectively. The observations and simulations (filled circles) are well fitted by the sum (solid line) of a scaled version of the power spectrum of the point spread function and a constant (dashed lines). We note that $R-I$ is generally small $(\approx 0.5)$ for early-type dwarfs and thus the results from the two filters comparable.

1998,Trentham \& Hodgkin 2002) and Fornax (Kambas et al. 2000, Hilker et al. 2003) showed that the ambiguity in attributing membership status to cluster/group galaxy candiates is the ultimate source of uncertainty on the quest to find the accurate shape, slope, and possible turning point of the faint end of the galaxy luminosity function. To achieve an undisputable separation between members of an environment and background/foreground objects distances are needed.

That is why we are currently exploring the possibility to verify membership of all known 232 group candidates of the NGC1407 Group (Trentham \& Tully 2002) by means of measuring SBF distances using high quality images from Subaru, CFHT, and HST. This galaxy group at $\approx 25 \mathrm{Mpc}$ is quite noteworthy. Dense knots of early-type galaxies are found across a considerable scale range from rich clusters with in excess of $10^{15} M_{\odot}$ to just a few big galaxies in a halo of $10^{13} M_{\odot}$ (Tully 2005). Within the domain $V<3000 \mathrm{~km} \mathrm{~s}^{-1}$, the NGC 1407 Group is the best case at the low mass end of this range of E/S0 knots. It contains only two $L^{\star}$ galaxies, yet the 1 -D velocity dispersion is with $385 \mathrm{~km} \mathrm{~s}^{-1}$ comparable to the Fornax cluster. It contains many dE dwarfs. The goal of our extensive SBF study is to obtain a complete inventory.

Subaru and CFHT images can provide SBF signals satisfactory for distance estimates for the 63 NGC1407 Group candidates that are brighter than $M_{R} \sim-14$ mag. Examples of the power spectra obtained for two galaxies are shown in the top panels of Fig. 3 . The remaining 169 low luminous, low-surface brightness dwarfs previously unaccessible to distance and redshift measurements, will be target objects in our HST SNAP program. Simulated power spectra for dwarfs as faint as $M_{I}=-11.5 \mathrm{mag}$ are shown in the lower 
panels of Fig. 3. The fluctuation signal remains above the noise level out to wave number $k \sim 15$. Indeed, the SBF detection with HST/ACS of a dwarf with $M_{I}=-11.5 \mathrm{mag}$ will be better than the SBF detection with the current Subaru data of the galaxy with $M_{R}=-16.5$ mag. Based on these simulation predictions we will comfortably be able to measure SBF signals and distances for our faintest candidates.

The results from this SBF study will enable us to establish a distance-based galaxy luminosity function for that particular group environment down to $M_{R}=-11$ mag. Moreover, the feedback from the NGC1407 Group will then be combined with spectroscopic and neutral hydrogen data measured for the few late-type group member candidates to allocate accurate membership probabilities to the morphology-based five-staged rating scheme employed by Trentham \& Tully (2002). Transferring the calibrated rating scheme to other galaxy groups under study will then help to much better constrain the luminosity functions found in other environments, permitting progress on the important question of whether there are differences with environment.

\section{Conclusion}

With the brief discussion of some recent projects and results based on SBF distances of early-type dwarf galaxies I hope I was able to let readers discover the great potential of this powerful dE distance indicator. Due to its simplicity and efficiency there is little doubt that this method will play an important role in the future 3 -D surveying of the local universe, particularly taking advantage of the deep, high quality CCD imaging data produced by the steadily increasing number of wide-field cameras on present and new generation telescopes.

\section{Acknowledgements}

HJ would like to express his gratitude to the IAU for the financial support of this most fruitful and enjoyable meeting. Part of my research on dwarf galaxies is supported by the ARC Discovery Grant DP0343156.

\section{References}

Arnaboldi, M., et al. 2002, AJ 123, 760

Barazza, F.D., Binggeli, B. \& Jerjen, H. 2002, A\& $A$ 391, 823

Barnes, D.G., et al. 2001, MNRAS 322, 486

Bertelli, G., et al. 1994, A\&SAS 106, 275

Binggeli, B. \& Cameron, L.M. 1991, A\&A 252, 27

Binggeli, B. \& Jerjen, H. 1998, A\&A 333, 17

Binggeli, B., Sandage, A. \& Tammann, G.A. 1988, ARAA 26, 509

Bode, P., Ostriker, J.P. \& Turok, M. 2001, ApJ 556, 93

Carignan, C. \& Freeman, K.C. 1988, ApJ 332, 33

Côté, P. et al. this volume

Dekel, A. \& Silk, J. 1986, ApJ 303, 39

Doyle, M. et al. 2005, MNRAS, 361, 34

Dunn, L. \& Jerjen, H. this volume

Ferguson, A., Gallagher, J. \& Wyse, R. 2000, AJ 120, 821

Gnedin, N.Y. 2000, ApJ 542, 535

Grebel, E.K. 2001, Astrophysics and Space Science Supplement 277, 231

Haiman, Z., Thoul, A.A. \& Loeb, A. 1996, ApJ 464, 523

Harris, W.E., Durrell, P.R., Pierce, M.J. \& Secker, J. 1998, Nature 395, 45

Hilker, M., Mieske, S. \& Infante, L. 2003, A $\& A$ 397, L9 
Jerjen, H., Binggeli, B. \& Barazza, F. 2004, AJ 127, 771

Jerjen, H., Freeman, K.C. \& Binggeli, B. 1998, AJ 116, 2873

Jerjen, H., Freeman, K.C. \& Binggeli, B. 2000, AJ 119, 166

Jerjen, H., Kalnajs, A. \& Binggeli, B. 2000, A $\& A 358,845$

Jerjen, H. et al. 2001, A\&SA 380,90

Kambas, A. et al. 2000, AJ 120, 1316

Karachentsev, I.D. et al. 1999, A\&SA 352, 399

Karachentsev, I.D. et al. 2000, $A \& A 363,117$

Karachentsev, et al. 2004, AJ 127, 2031

Kilborn., V. et al. 2000, AJ 120, 1342

Klypin, A. et al. 1999, ApJ 522, 82

Koribalski, B.S. et al. 2004, AJ 128, 16

Krumm, N. \& Burstein, D. 1984, AJ 89, 1319

Mao, S. \& Mo, H.J. 1998, MNRAS 296, 847

Mastropietro, C. et al. 2005, astro-ph/0411648

Mei, S. et al. 2005, ApJ 625, 121

Mieske, S. \& Hilker, M. 2003, A\&SA 410, 445

Moore, B., Lake, G. \& Katz, N. 1998, ApJ 495, 139

Moore, B. et al. 1999, ApJ 524, L19

Neilsen, E.H., Jr. \& Tsvetanov, Z.I. 2000, ApJ 536, 255

Oosterloo, T. et al. IAU Symposium No. 217 (San Francisco: Astronomical Society of the Pacific), p. 48

Phillipps, S. et al. 1998, ApJ, 493, L59

Rekola, R., Jerjen, H. \& Flynn, C. 2005, A\& A 437, 823

Ryder, S.D. et al. 2001, ApJ 555, 232

Spergel, D.N. \& Steinhardt, P.J. 2000, Phys Rev Lett 84, 3760

Thoul, A.A. \& Weinberg, D.H. 1996, ApJ 465, 608

Tonry, J.L. et al. 1997, ApJ 475, 399

Tonry, J.L. et al. 2001, ApJ 546, 681

Tonry, J.L. \& Schneider, D.P. 1988, AJ 96, 807

Trentham, N. \& Hodgkins, S. 2002, MNRAS 333, 423

Trentham, N. \& Tully, R.B. 2002, MNRAS 335, 712

Tully, R.B. 2005, ApJ 618, 214

van den Bergh, S. 1972 ApJ 171, L31

Warren, B. E., Jerjen, H. \& Koribalski, B. S. 2004, AJ 128, 1152

West, M.J. \& Blakeslee, J.P. 2000, ApJ 543, L27

Worthey, G. 1994, ApJS 95, 107

\section{Discussion}

Nelson Caldwell: It's interesting that the dwarfs you have observed so far fall either on the linear or parabolic part of the $\bar{m}$ vs. B-R relation. Naively, one might expect a range of star formation histories in galaxies and so a scattering of points between the two curves. With more objects observed, do you think the dEs will fill in the area between the curves?

Helmut JerJen: A possible explanation for this empty region is that the colour of the RGB is largely independent of age but is strongly dependent on metallicity (Fig. 1 of Da Costa 1997) and that for a fixed metallicity the colour of the HB has a discontinuity from blue for the oldest populations $(\sim 15 \mathrm{Gyr})$ to red at younger ages ( $\leqslant 10 \mathrm{Gyr}$; Fig. 4 of Da Costa 1997). The latter effect is more marked for lower metallicities. These two trends describe the behavior of $(B-R)$ and $\bar{M}_{R}$ in Fig. 1 . In the models, only the very old and metal-poor populations are located on the parabolic curve. With an increasing fraction 
of younger stars the total population gets redder and the model values reach quickly the linear curve.

Steffen Mieske: How do you know that the models you are using for the SBF calibration are correct?

Helmut JerJen: In Jerjen, Freeman \& Binggeli (1998) we compared the Worthey+Yale and Worthey+Padua results with empirical data and found much better agreements with the latter models. This good agreement still exists after using the latest SBF data for seven calibration galaxies shown in Fig. 1. But ultimately we are aiming for a modelindependent SBF calibration based solely on nearby dEs.

IgOR Chilingarian: For the color-metallicity relation you assume old ages (>8 Gyr), but in the paper of Geha et al. who observed several tens of dEs in Virgo the mean age is estimated 3 Gyr (using Lick indices). How will this affect the relation?

Helmut JerJen: The $(B-R, \bar{M})$ data points for stellar populations of younger ages $(<8 \mathrm{Gyr})$ would move down the linear branch in Fig. 1 blending with the results for older stellar populations but lower metallicities. Caldwell, Rose \& Concannon (2003) also tried to disentangle the effects of age and metallicity in a sample of Virgo earlytype galaxies. The Balmer lines in these dwarfs were interpreted within the Lick index system as being primarily caused by young age, rather than by low metallicity leading to galaxy ages ranged from 1-16 Gyr. This interpretation stands in contrast to Maraston \& Thomas $(2000,2001)$, who showed that a mix of an old metal-rich and an old metalpoor component can produce strong Balmer and metal lines without invoking a young population. I also would like to recall another result from age-sensitive narrow-band photometry in the modified Strömgren filter system by Rakos et al. (2001). These authors derived a mean age of $10 \pm 1$ Gyr for a sample of 27 Fornax dEs.

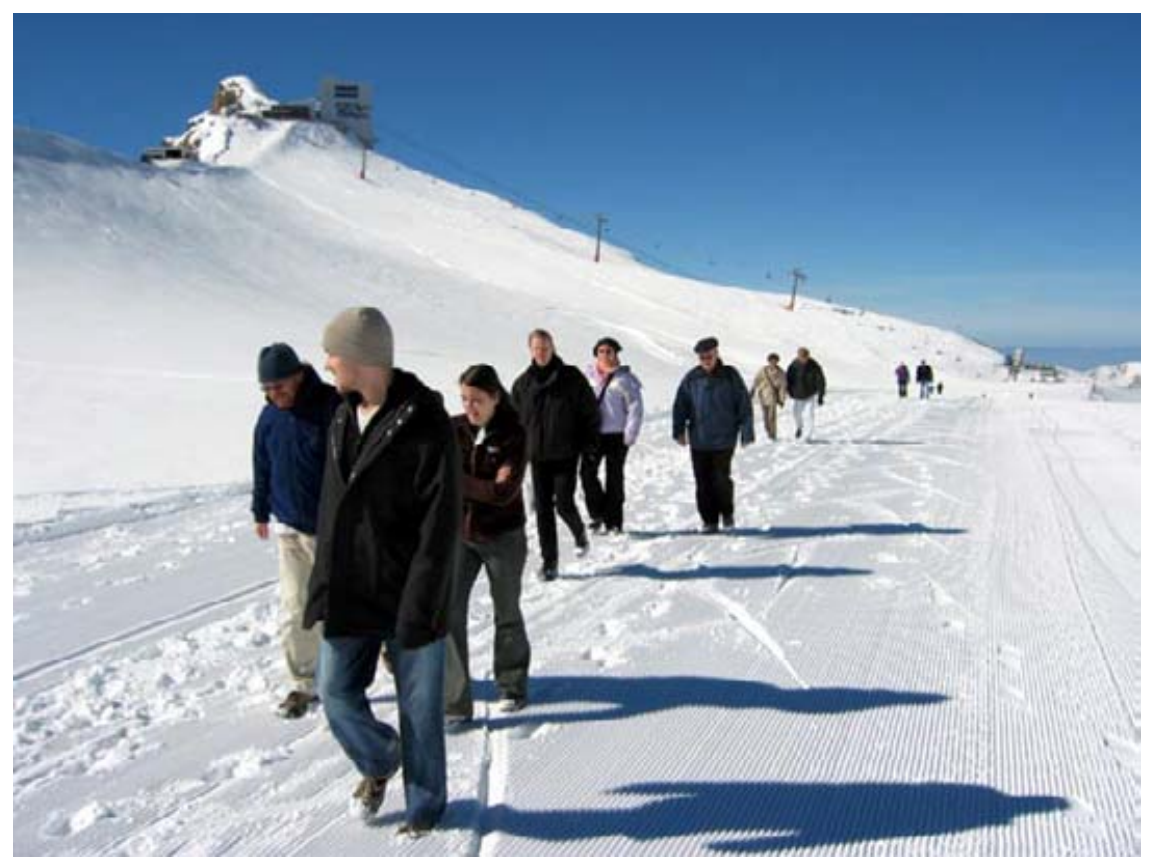

